\title{
Overscreening Diamagnetism in Cylindrical Superconductor-normal Metal-heterostructures
}

\author{
W. Belzig, ${ }^{1}$ C. Bruder, ${ }^{2}$ and Yu.V. Nazarov ${ }^{3}$ \\ ${ }^{1}$ Department of Physics, University of Konstanz, 78457 Konstanz, Germany \\ ${ }^{2}$ Department of Physics and Astronomy, University of Basel, Klingelbergstrasse 82, 4056 \\ Basel, Switzerland \\ E-mail: Christoph.Bruder@uni.bas.ch \\ ${ }^{3}$ Kavli Institute of NanoScience, Delft University of Technology, 2628 CJ Delft,
}

The Netherlands

We study the linear diamagnetic response of a superconducting cylinder coated by a normal-metal layer due to the proximity effect using the clean limit quasiclassical Eilenberger equations. We compare the results for the susceptibility with those for a planar geometry. Interestingly, for $R \sim d$ the cylinder exhibits a stronger overscreening of the magnetic field, i.e., at the interface to the superconductor it can be less than $(-1 / 2)$ of the applied field. Even for $R \gg d$, the diamagnetism can be increased as compared to the planar case, viz. the magnetic susceptibility $4 \pi \chi$ becomes smaller than $-3 / 4$. This behavior can be explained by an intriguing spatial oscillation of the magnetic field in the normal layer.

\section{INTRODUCTION}

A normal metal in electronic contact to a superconductor acquires superconducting properties. This phenomenon is called proximity effect. ${ }^{1,2}$ One of these properties is the diamagnetic screening of an applied magnetic field, which has been studied in a series of experimental ${ }^{3-8}$ and theoretical works. ${ }^{9-14}$

First predictions on the induced screening properties were made on the basis of the Landau-Ginzburg theory by de Gennes and co-workers. ${ }^{1,2}$ The proximity effect is governed by the thermal coherence length $\xi_{T}$, given by $\sqrt{D / T}$ in the diffusive regime and $v_{\mathrm{F}} / T$ in the clean limit. Here $v_{\mathrm{F}}$ denotes the Fermi velocity and $D$ the diffusion coefficient inside the normal metal. Throughout the paper, we will set $\hbar=k_{\mathrm{B}}=1$. The Ginzburg-Landau approach is valid outside the mesoscopic regime in which 
$\xi_{T} \ll d$, where $d$ is the thickness of the normal metal film. In this approach a region of width $\sim \xi_{T}$ of the normal metal screens the magnetic field and consequently the susceptibility of the normal metal $4 \pi \chi=4 \pi M / H=$ $-\xi_{T} / d$ increases with the coherence length. Strictly speaking the approach of de Gennes et al. requires in addition a local constitutive relation $j(x) \sim$ $A(x),{ }^{15}$ which is only the case under special circumstances. ${ }^{14}$ The first experimental studies were in agreement with these early predictions ${ }^{16,17}$ and the predicted screening was observed. However, in this experiment the mesoscopic regime was not reached. According to the prediction the susceptibility approaches almost the ideal value $4 \pi \chi=-1$.

In 1980, Zaikin investigated the screening properties of clean normal metals in the clean limit. In that case, the constitutive relation is complete non-local, viz. $j \sim \int_{0}^{d} A(x) d x$. This has the interesting consequence that the susceptibility saturates at low temperatures at $75 \%$ of the ideal diamagnetic value. The magnetic field inside the normal metal decays linearly at low temperatures and even changes sign to reach a maximal opposite field of $-H / 2$ at the interface to the superconductor. Note, that a similar effect occurs in clean type-I superconductors. ${ }^{18}$

Around the same time a series of experiments by Mota and co-workers found an interesting low-temperature anomaly in the magnetic response of cylindrical structures. ${ }^{3,5-7,19,20}$ At very low temperatures the diamagnetic signal decreased again and in the end became even paramagnetic. This socalled reentrant effect of the magnetic susceptibility has triggered a number of theoretical explanations, ${ }^{21-24}$ however a final experimental verification of one proposal is still missing.

The effect of elastic impurity scattering was the subject of a number of works. A finite elastic mean free path reduces the range of the currentfield relation and changes the screening properties drastically. ${ }^{14}$ Remarkably, impurities can enhance the screening ability at some temperatures and even have an effect if the mean free path exceeds the thickness of the normal layer. This quasiclassical description ${ }^{25}$ was shown to agree with the experimental data for temperatures above the reentrance regime. ${ }^{26}$ In a number of other works the non-linear magnetic properties have been discussed numerically ${ }^{12}$ and analytically. ${ }^{13}$ Other works addressed the effect of a non-ideal interface. ${ }^{27-30}$

In this work, we investigate the effect of a cylindrical geometry on the magnetic screening properties. A sketch of the system is shown in the left panel of Fig. 1. We will consider a superconducting cylinder covered by a normal-metal layer. The superconducting core has radius $R$; the normal-metal layer has thickness $d$, i.e., the total radius of the cylindrical sample is $R+d$. Both the superconductor and the normal metal are 


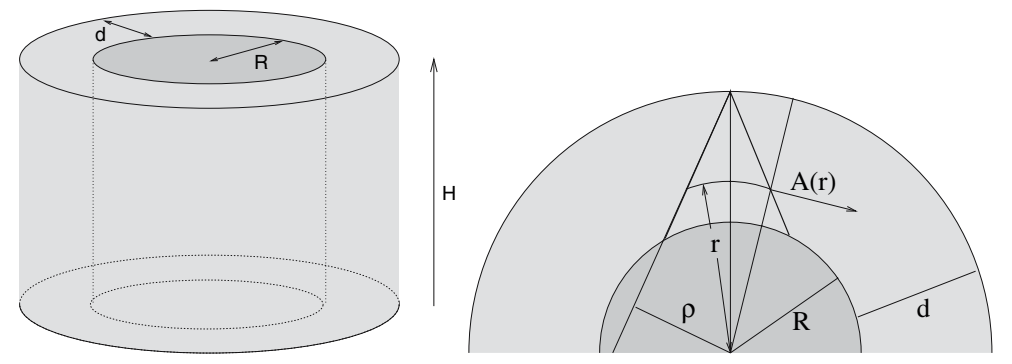

Fig. 1. Left panel: a superconducting cylinder surrounded by a normal-metal layer. A magnetic field is applied in parallel to the axis of the cylinder. Right panel: parameterization of the averaging procedure over the Fermi surface.

assumed to be in the clean limit. A magnetic field is applied parallel to the axis of the cylinder, and the task is to calculate the magnetic susceptibility of the normal-metal layer (we assume an ideal diamagnetism of the superconductor).

We assume the pair potential $\Delta$ to have a step-like dependence on the radial coordinate,

$$
\Delta(r)=\Delta \Theta(R-r)
$$

and we will neglect self-consistency. This is justified since the superconductor is assumed to be much thicker than the coherence length and the magnetic field is assumed to be much less than the critical field. A small suppression of $\Delta$ at the interface would not lead to qualitative changes.

Throughout this paper, we will use the symmetric gauge for the vector potential

$$
\boldsymbol{A}(\boldsymbol{r})=A(r) \boldsymbol{e}_{\varphi}, \quad \boldsymbol{H}(\boldsymbol{r})=H \boldsymbol{e}_{z} \Theta(r-R-d) .
$$

Here $\boldsymbol{e}_{\varphi}$ is the unit vector in direction of $\varphi$ and $\boldsymbol{e}_{z}$ is the unit vector in $z$-direction.

The rest of the paper is organized as follows: in Sect. 2, we introduce the formalism of quasiclassical Green's functions and discuss how to solve the Eilenberger equation. Its solution leads to an expression for the supercurrent density in the normal layer, that is combined with Maxwell's equation in Sect. 3 which results in an integro-differential equation for the vector potential. We solve this equation numerically in Sect. 4 and calculate the magnetic susceptibility of the normal layer. In the planar limit, the results agree with earlier work, whereas in the cylindrical case we find new and interesting screening behavior and a non-trivial oscillating field distribution. 


\section{SOLUTION OF THE QUASICLASSICAL EQUATIONS}

The goal is to solve the clean-limit Eilenberger equation ${ }^{31,32}$

$$
-\boldsymbol{v}_{\mathrm{F}} \boldsymbol{\partial} \hat{g}_{\omega}\left(r, \boldsymbol{v}_{\mathrm{F}}\right)=\left[\left(\omega+i e \boldsymbol{v}_{\mathrm{F}} \boldsymbol{A}(r)\right) \hat{\tau}_{3}+\hat{\Delta}(r), \hat{g}_{\omega}\left(r, \boldsymbol{v}_{\mathrm{F}}\right)\right]
$$

in the cylindrical geometry described above. Here $\omega=\pi T(2 n+1)$ denotes a Matsubara frequency and $\hat{\Delta}=\Delta \hat{\tau}_{1}$ is the off-diagonal pair potential of the superconductor. We will assume the following boundary conditions: a fully transparent boundary at the NS interface,

$$
\hat{g}_{\omega}^{s}\left(R-0, \boldsymbol{v}_{\mathrm{F}}\right)=\hat{g}_{\omega}^{n}\left(R+0, \boldsymbol{v}_{\mathrm{F}}\right)
$$

and specular reflection at the outer boundary

$$
\hat{g}_{\omega}^{n}\left(R+d, \boldsymbol{v}_{\perp}, \boldsymbol{v}_{\|}\right)=\hat{g}_{\omega}^{n}\left(R+d,-\boldsymbol{v}_{\perp}, \boldsymbol{v}_{\|}\right) .
$$

Deep in the superconductor, we have

$$
\hat{g} \rightarrow \frac{1}{\Omega}\left(\begin{array}{cc}
\omega & \Delta \\
\Delta & -\omega
\end{array}\right),
$$

where $\Omega=\sqrt{\Delta^{2}+\omega^{2}}$. These equations have to be solved along classical trajectories, along which they become effectively one-dimensional. The general solution is a superposition of the homogeneous solution $\hat{g}_{h}$ and the rising resp. decaying solutions $\hat{g}_{+}$and $\hat{g}_{-}$. In the normal layer, $R \leq r \leq$ $R+d$ we make the Ansatz

$$
\hat{g}(r)^{n}=\alpha_{0} \hat{g}_{h}^{n}+\alpha_{+} \hat{g}_{+}^{n} e^{\eta(r-R)}+\alpha_{-} \hat{g}_{-}^{n} e^{-\eta(r-R)},
$$

where

$$
\eta(x)=\frac{2 \omega}{\left|v_{\mathrm{F} x}\right|} x+2 i e \frac{v_{\mathrm{F} y}}{\left|v_{\mathrm{F} x}\right|} \int_{0}^{x} A\left(R+x^{\prime}\right) d x^{\prime}
$$

is the complex phase accumulated along the trajectory and

$$
\hat{g}_{h}^{n}=\tau_{3}, \quad \hat{g}_{ \pm}^{n}=\frac{1}{2}\left(\tau_{1} \mp i \operatorname{sign}\left(v_{\mathrm{F} x}\right) \tau_{2}\right) .
$$

In the superconductor, the corresponding Ansatz reads

$$
\hat{g}(r)^{s}=\beta_{0} \hat{g}_{h}^{s}+\beta_{+} \hat{g}_{+}^{s} e^{\frac{2 \Omega(r-R)}{\left|v_{\mathrm{F} x}\right|}}+\beta_{-} \hat{g}_{-}^{s} e^{-\frac{2 \Omega(r-R)}{\left|v_{\mathrm{F} x}\right|}},
$$

where

$$
\hat{g}_{h}^{s}=\frac{1}{\Omega}\left(\omega \tau_{3}+\Delta \tau_{1}\right), \quad \hat{g}_{ \pm}^{s}=\frac{1}{2 \Omega}\left(\Delta \tau_{3}-\omega \tau_{1} \pm i \Omega \operatorname{sign}\left(v_{\mathrm{F} x}\right) \tau_{2}\right) .
$$


We have neglected the magnetic field in the superconductor, since the penetration depth is assumed to be much smaller than the coherence length. Furthermore, we will neglect the possibility that a quasiparticle trajectory leaves the superconducting core again. ditions

The coefficients $\alpha_{0}, \beta_{0}, \alpha_{+/-}$, and $\beta_{+/-}$fulfill the normalization con-

$$
\alpha_{0}^{2}+\alpha_{+} \alpha_{-}=1, \quad \beta_{0}^{2}+\beta_{+} \beta_{-}=1 .
$$

Using the boundary conditions, we find that

$$
\beta_{-}=0 \quad \text { and } \quad \beta_{0}=1
$$

deep in the superconductor, whereas the boundary condition at $x=R+d$ in the normal conductor leads to

$$
\alpha_{+} e^{\eta(d)}=\alpha_{-} e^{-\eta(d)} .
$$

The other two coefficients can be determined from the matching condition at $r=R$ leading to the final result

$$
\alpha_{0}=\frac{\Omega+\omega \operatorname{coth}(\eta(d))}{\omega+\Omega \operatorname{coth}(\eta(d))}, \quad \beta_{+}=\frac{2 \Delta}{\omega+\Omega \operatorname{coth}(\eta(d))} .
$$

The diagonal component of the Green's function in the normal metal ( $\tau_{3}$-component of $\hat{g}^{n}$ ) is constant along a trajectory,

$$
g(\mathcal{L})=\frac{\Omega+\omega \operatorname{coth}(\eta(d))}{\omega+\Omega \operatorname{coth}(\eta(d))},
$$

where

$$
\eta=\frac{\omega L_{\mathcal{L}}}{v_{\mathrm{F}}}+i e \int_{\mathcal{L}} \boldsymbol{A}(\boldsymbol{r}) \boldsymbol{d} \boldsymbol{r}
$$

and

$$
\Omega=\sqrt{\Delta^{2}+\omega^{2}} .
$$

Here, $L_{\mathcal{L}}$ is the length of trajectory $\mathcal{L}$ and $\int_{\mathcal{L}}$ the line integral along the trajectory. Here we have generalized our previous formula to the more general cylindrical case. In the planar limit $R / d \rightarrow \infty$, our solution agrees with earlier work. ${ }^{9}$

This solution determines the screening current in the normal metal layer via

$$
\boldsymbol{j}(\boldsymbol{r})=-i 4 \pi e N_{0} T \sum_{\omega>0}\left\langle\boldsymbol{v}_{\mathrm{F}} \operatorname{Tr} \hat{\tau}_{3} \hat{g}_{\omega}\left(\boldsymbol{v}_{\mathrm{F}}\right)\right\rangle=-i 8 \pi e N_{0} T \sum_{\omega>0}\left\langle\boldsymbol{v}_{\mathrm{F}} \alpha_{0}\right\rangle .
$$


Here, $N_{0}$ denotes the density of states at the Fermi energy. We note, that the screening current is determined by the diagonal component of $\hat{g}$ only, which for a given trajectory is constant along that particular trajectory. However, the angular average may induce a space dependence, since certain trajectories, which do not hit the superconductor have to be excluded from the angular average. This will be important for the cylindrical geometry considered below.

\section{SOLUTION OF MAXWELL'S EQUATION}

We will solve Maxwell's equation to obtain the spatial dependence of the magnetic field in the normal metal from the screening-current density. In the planar limit $R / d \rightarrow \infty$, it is possible to give an analytical solution, which we discuss briefly. ${ }^{9}$ In this case, the current density in the normal layer turns out to be constant as a function of the spatial coordinates,

$$
\begin{aligned}
& j \stackrel{R / d \rightarrow \infty}{=} 16 e N_{0} v_{\mathrm{F}} T \sum_{\mu=0}^{\infty} \int_{0}^{\frac{\pi}{2}} d \theta \int_{0}^{\frac{\pi}{2}} d \varphi \sin ^{2} \theta \cos \varphi \\
& \times \frac{\Delta^{2}(\cosh \Phi+\cos \phi) \sin \phi}{[\omega \sinh \Phi+\Omega(\cosh \Phi+\cos \phi)]^{2}} .
\end{aligned}
$$

Here,

$$
\Phi=\frac{2 \omega L}{v_{\mathrm{F}}}=\frac{4 \omega d}{v_{\mathrm{F}} \cos \theta}
$$

is an effective trajectory length, and

$$
\phi=4 \tan \theta \cos \varphi e \int_{R}^{R+d} A(r) d r
$$

is the Aharonov-Bohm phase along this trajectory. The current density depends on temperature and applied magnetic field $H$. More precisely, there is a non-local relationship between current density and vector potential that can be understood in the following way: consider the semiclassical trajectory of an electron in the normal metal starting at the NS interface with velocity $\boldsymbol{v}_{\mathrm{F}}$. It will be specularly reflected at the outer interface and eventually hit the superconductor, see the right panel of Fig. 1. There it will be Andreev-reflected and return as a hole along the trajectory that the electron took before. Such semiclassical closed orbits correspond to quantum bound states and are known as Andreev levels. ${ }^{33}$ In the presence of a vector potential, the electron picks up a phase 


$$
e \int_{L} \boldsymbol{A}(\boldsymbol{r}) \boldsymbol{d} \boldsymbol{r}=2 e \frac{v_{\mathrm{F} \varphi}}{v_{\mathrm{F} r}} \int_{R}^{R+d} A(r) d r,
$$

which will lead to an energy shift for the Andreev levels ${ }^{34}$

$$
\frac{e v_{\mathrm{F} \varphi}}{d} \int_{R}^{R+d} A(r) d r .
$$

Since the Andreev levels are homogeneous in the normal layer, this energy shift leads to a spatially constant current density.

Solving Maxwell's equation with the boundary conditions

$$
A(R)=0
$$

and

$$
\left.\frac{d}{d r} A(r)\right|_{r=R+d}+\frac{A(R+d)}{R+d}=H
$$

leads to the following $z$-component of the magnetic field:

$$
\begin{aligned}
B(r)=H-4 \pi j(T, H)(R+d-r) & \text { for } R<r<R+d, \\
B(r)=H & \text { for } r>R+d .
\end{aligned}
$$

The field may change its sign within the normal-metal layer if

$$
4 \pi j(T, H) d>H \text {. }
$$

This "overscreening" effect was first discussed by Zaikin. ${ }^{9}$ A numerical evaluation of the current density Eq. (20) at all temperatures provides a solution of Maxwell's equations for arbitrary fields.

For a cylindrical geometry, the current density in the normal layer depends on the radial coordinate. The $\varphi$-component of the current density reads

$$
j(r)=-i e N_{0} T \sum_{\omega>0}\left\langle v_{\mathrm{F} \varphi} \alpha_{0}\right\rangle(r) .
$$

The average over the Fermi surface can be parameterized in a way that allows to easily exclude trajectories that do not meet the superconductor, i.e., do not contribute to the current. Instead of performing the usual angular average

$$
\int \frac{d \Omega_{v_{\mathrm{F}}}}{4 \pi} \cdots=\int_{-1}^{1} \frac{d \cos \theta}{2} \int_{0}^{2 \pi} \frac{d \varphi}{2 \pi} \ldots
$$


it is convenient to define $\rho=r \sin \varphi$ which leads to

$$
\int_{-r}^{r} \frac{d \rho \rho}{r \sqrt{r^{2}-\rho^{2}}} \int_{-1}^{1} \frac{d \cos \theta}{4 \pi} \cdots
$$

see the right panel of Fig. 1 for the geometrical interpretation of $\rho$. Obviously, only those trajectories contribute for which $|\rho|<R$. Such a trajectory has length

$$
L(\rho, \theta)=2 \frac{\sqrt{(R+d)^{2}-\rho^{2}}-\sqrt{R^{2}-\rho^{2}}}{|\sin \theta|} .
$$

Using this parameterization, the line integral over the vector potential can be written as

$$
\int \boldsymbol{A}(r) \boldsymbol{d} \boldsymbol{x}=2 \int_{R}^{R+d} d r^{\prime} A\left(r^{\prime}\right) \frac{\rho}{\sqrt{r^{\prime 2}-\rho^{2}}} \sin \theta .
$$

We finally obtain the following expression for the current density, Eq. (29),

$$
\begin{aligned}
j(r)= & 4 e N_{0} v_{\mathrm{F}} T \sum_{\omega>0} \int_{0}^{R} d \rho \int_{0}^{\pi / 2} d \theta \frac{\rho \sin ^{2} \theta}{2 \pi r \sqrt{r^{2}-\rho^{2}}} \\
& \times \frac{\Delta^{2} \sin \phi(\cosh \Phi+\cos \phi)}{(\omega \sinh \Phi+\Omega(\cosh \Phi+\cos \phi))^{2}+\omega^{2} \sin ^{2} \phi},
\end{aligned}
$$

where

$$
\Phi=\frac{2 \omega L(\rho, \theta)}{v_{\mathrm{F}}}
$$

and

$$
\phi=4 e \int_{R}^{R+d} d r^{\prime} A\left(r^{\prime}\right) \frac{\rho}{\sqrt{r^{\prime 2}-\rho^{2}}} .
$$

Using Maxwell's equations, and to linear order in the magnetic field, we find the following linear integro-differential equation for the vector potential:

$$
\begin{aligned}
& \xi^{2} \frac{d}{d r} \frac{1}{r} \frac{d}{d r} r A(r)=K \frac{T}{T_{c}} \sum_{\omega>0} \int_{0}^{R} \frac{d \rho}{\xi} \int_{0}^{\pi / 2} d \theta \\
& \times \frac{\rho^{2} \sin ^{2} \theta}{r \sqrt{r^{2}-\rho^{2}}} \frac{\Delta^{2}(\cosh \Phi+1)}{(\omega \sinh \Phi+\Omega(\cosh \Phi+1))^{2}} \int_{R}^{R+d} \frac{A\left(r^{\prime}\right) d r^{\prime}}{\sqrt{r^{\prime 2}-\rho^{2}}}
\end{aligned}
$$


Here, the effective length of the trajectories is given by

$$
\Phi=\frac{4 \omega\left(\sqrt{(R+d)^{2}-\rho^{2}}-\sqrt{R^{2}-\rho^{2}}\right)}{v_{\mathrm{F}}|\sin \theta|} .
$$

We have introduced dimensionless units; lengths are measured in units of the (clean-limit) coherence length at $T_{c}$

$$
\xi=\frac{v_{\mathrm{F}}}{2 \pi T_{c}}
$$

the current density contains a dimensionless material constant,

$$
K=32 e^{2} N_{0} v_{\mathrm{F}}^{2} \xi^{2}=\frac{24}{\pi}\left(\frac{\xi}{\lambda_{N}}\right)^{2},
$$

where $\lambda_{N}=\left(4 \pi e^{2} n_{e} / m\right)^{-1 / 2}$ is a length scale that is defined in analogy with the London penetration depth, but using the electron density $n_{e}$ of the normal metal instead of the superfluid density of the superconductor.

\section{RESULTS FOR THE MAGNETIC SUSCEPTIBILITY}

We will now discuss the susceptibility in the linear-response regime for the planar and cylindrical case.

The numerical solution in the planar case for $K=10$ and a normallayer thickness of $20 \xi$ leads to the current densities and magnetic fields shown in Fig. 2. The left panel shows the current density (that is constant for the planar case). The right panel shows the corresponding spatially dependent magnetic fields. For $T \lesssim 0.1 T_{c}$ the condition (28) is fulfilled, and the field changes sign at the NS interface. Below $T=0.05 T_{c}$ the current density does not increase any more, and we only show the curve for $T=$ $0.01 T_{c}$.

As discussed before, the current density depends on the spatial coordinate in the cylindrical case. Figure 3 shows the current density and the magnetic field for $K=10$, both the radius $R$ of the superconductor and the normal-layer thickness have been chosen as $20 \xi$. The local current density can be significantly larger than in the planar case, e.g., three times as large at the NS interface for $T \rightarrow 0$. The screening effect is weaker than in the planar case close to the outer interface; close to the NS-interface it is increased, and the overscreening effect may be even larger than in the planar case. 

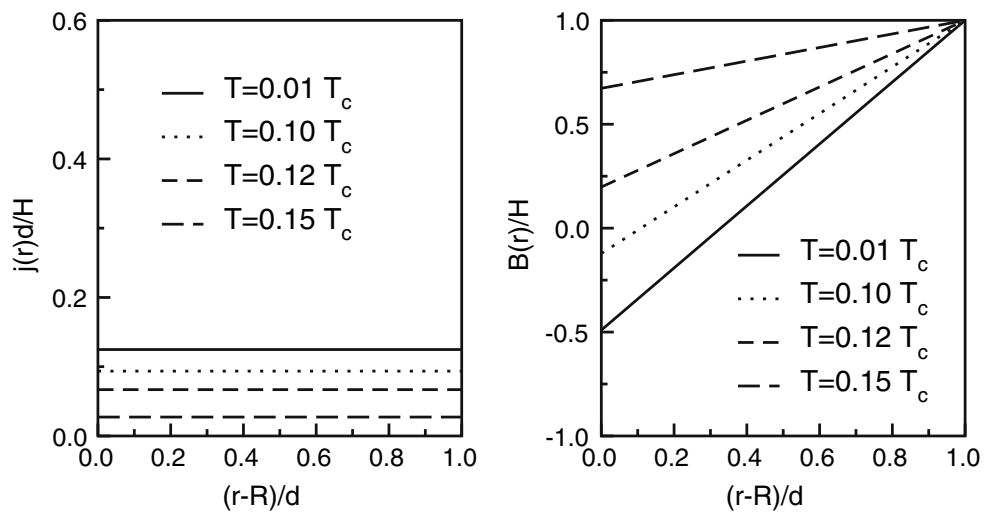

Fig. 2. Current density (left panel) and magnetic field (right panel) for a planar geometry with $\xi=d / 20, R / d \rightarrow \infty, K=10$, and different temperatures. The material parameter $K$ is defined in Eq. (40).
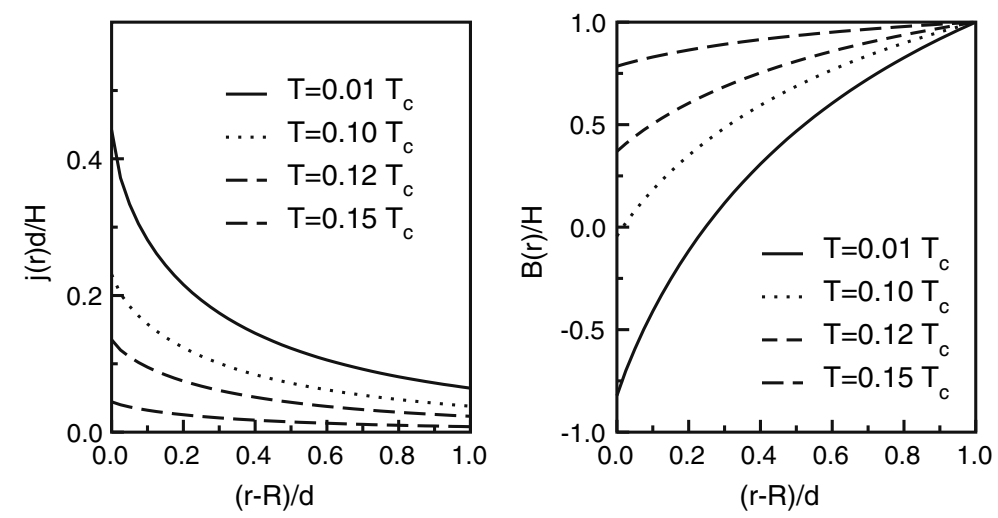

Fig. 3. Current density (left panel) and magnetic field (right panel) for a cylindrical geometry with $d=R, \xi=d / 20, K=10$, and different temperatures.

The susceptibility of the normal-metal layer can be obtained from the solution of Maxwell's equation via

$$
4 \pi \chi(T, H)=\frac{A(R+d)}{A_{e}(R+d)}-1,
$$

where $A_{e}(r)$ is the vector potential in the absence of the normal layer. In the planar case, the vector potential can be calculated analytically and leads to

$$
4 \pi \chi(T, H)=-2 \pi \frac{j(T, H) d}{H} .
$$


We will now give explicit expressions for the susceptibility in the linearresponse regime for the planar and cylindrical case.

In the planar case, Eq. (20) can be simplified assuming $e \int_{R}^{R+d} A(r) d r \ll$ 1 or $H \ll \Phi_{0} / d^{2}$. In other words, the flux enclosed by a semiclassical trajectory is less than a flux quantum $\Phi_{0}$; the phase picked up by the electron has to be less than $2 \pi$. For experimentally relevant normal-layer thicknesses of $d \approx 1 \mu \mathrm{m}$ these conditions are fulfilled for fields $H \leq 20 \mathrm{G}$. The current density (20) factorizes like

$$
j(T, H)=-e j_{S}(T) \int_{R}^{R+d} A(r) d r,
$$

where

$$
\begin{aligned}
j_{S}(T)=4 \pi e N_{0} v_{\mathrm{F}} T \sum_{\mu=0}^{\infty} \int_{0}^{\frac{\pi}{2}} d \theta \sin ^{2} \theta \tan \theta \\
\frac{\Delta^{2}\left(\cosh \left(\frac{4 \omega d}{v_{\mathrm{F}} \cos \theta}\right)+1\right)}{\left[\omega \sinh \left(\frac{4 \omega d}{v_{\mathrm{F}} \cos \theta}\right)+\Omega\left(\cosh \left(\frac{4 \omega d}{v_{\mathrm{F}} \cos \theta}\right)+1\right)\right]^{2}} .
\end{aligned}
$$

The analytical solution of Maxwell's equation, Eq. (27) leads to the following expression for the susceptibility

$$
4 \pi \chi(T)=\frac{-3 \pi e j_{s}(T) d^{3}}{3+4 \pi e j_{S}(T) d^{3}} .
$$

In general, $j_{s}$ can be calculated only numerically. However, for $T=0$ an approximate analytical expression can be obtained,

$$
j_{S}(T=0) \approx \frac{2}{\pi^{3} e d \lambda_{N}^{2}} .
$$

For $d \gg \lambda_{N}$, Eq. (45) leads to a susceptibility that it is independent of geometry,

$$
4 \pi \chi(T=0)=-\frac{3}{4} .
$$

Field expulsion is never complete; the maximal value that the susceptibility can reach at any temperature is $3 / 4$ of an ideal diamagnet. ${ }^{9}$

Figure 4 shows the susceptibilities for different normal-layer thicknesses. In thicker layers, field expulsion occurs only at lower temperatures. The thickest layer reaches the saturation value given in Eq. (47) for $T \rightarrow 0$, since it fulfills $d \gg \lambda_{N}$. 


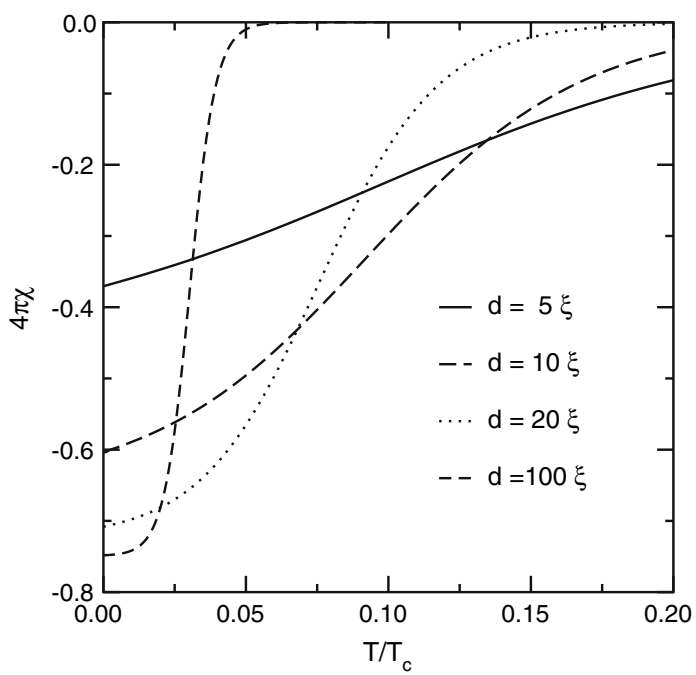

Fig. 4. Susceptibility of the normal-metal layer as a function of its thickness $d$ for $R / d \rightarrow \infty$ and $K=1$.

For the cylindrical case, the integro-differential equation for the vector potential, Eq. (37), has to be solved numerically.

Surprisingly, at low temperatures and for $K \gg 1$, the susceptibility does not go to $-3 / 4$. To analyze this parameter range in a quantitative way, we start from Eq. (37). We assume $d \ll R$ and concentrate on the contribution of the (long) trajectories with $R-\rho \simeq d$. The typical length of these trajectories is $L \simeq \sqrt{d R}$. They become important at low temperatures $T \ll v_{\mathrm{F}} / \sqrt{d R}$. We will assume the zero-temperature limit and replace the sum over $\omega$ in Eq. (37) by an integral. This integral converges at $\omega \simeq$ $v_{\mathrm{F}} / L \ll \Delta$, and the integration is readily performed. The next step is to implement the condition $d \ll R$. To this end, we do the following substitutions:

$$
\rho=R-\mu d
$$

and

$$
r=R+z d, \quad r^{\prime}=R+z^{\prime} d,
$$

where $\mu, z, z^{\prime}$ are dimensionless variables with $\mu>0$ and $0<z, z^{\prime}<1$. For instance, this gives $\sqrt{r^{\prime} 2-\rho^{2}} \rightarrow \sqrt{2 d R} \sqrt{z^{\prime}+\mu}$. Here and in the following, we will write $A(z)$ instead of $A(R+z d)$. After this substitution, Eq. (37) 
takes the following form:

$$
\left(\frac{\xi}{d}\right)^{2} \frac{d^{2} A}{d z^{2}}=\frac{K}{12 \sqrt{2}}\left(\frac{d}{R}\right)^{1 / 2} \int_{0}^{\infty} d \mu \int_{0}^{1} d z^{\prime} \frac{A\left(z^{\prime}\right)}{(\sqrt{\mu+1}-\sqrt{\mu}) \sqrt{z+\mu} \sqrt{z^{\prime}+\mu}} .
$$

There is a subtle point here: the integral over $\mu$ diverges at big $\mu$. Therefore, the right-hand side (r.h.s.) cannot describe the constant part of the current density correctly, however, it gives the correct inhomogeneous part. To get a physical result we perform the following renormalization procedure: we introduce a yet unknown constant $C$ on the r.h.s. to reproduce the constant part of the density and rewrite Eq. (50) as

$$
\begin{aligned}
\left(\frac{\xi}{d}\right)^{2} \quad & \frac{d^{2} A(z)}{d z^{2}}=C+\frac{K}{12 \sqrt{2}}\left(\frac{d}{R}\right)^{1 / 2} \int_{0}^{\infty} d \mu \int_{0}^{1} d z^{\prime} \\
& A\left(z^{\prime}\right)\left(\frac{1}{(\sqrt{\mu+1}-\sqrt{\mu}) \sqrt{z+\mu} \sqrt{z^{\prime}+\mu}}-4(\sqrt{\mu+1}-\sqrt{\mu})\right) .
\end{aligned}
$$

The $\mu$-dependent expression has been subtracted from the second term on the r.h.s. to assure that after subtraction the term gives zero if integrated over $z, z^{\prime}$. The integral over $\mu$ converges now. (We could use any other form of this subtraction that provides convergence of the integral since the change would be incorporated in $C$ ).

We do not have to know the coefficient that relates $C$ and $A$ provided that this coefficient is sufficiently big, which is true for $\lambda_{N} \ll d$. In this limit, $C$ has to be determined from the extra condition $\int d z A(z)=0$.

The remaining task is to numerically solve Eq. (51) under the conditions

$$
\begin{array}{r}
\int_{0}^{1} d z A(z)=0, \\
\left.\frac{d}{d z} A(z)\right|_{z=1}=H d, \\
A(0)=0 .
\end{array}
$$

The susceptibility is determined from this solution as

$$
4 \pi \chi=A(1) / B d-1 .
$$

Looking at Eq. (51) we see that the susceptibility is a function of the parameter

$$
\gamma \equiv 1 / \sqrt{K(d / \xi)^{2}(d / R)^{1 / 2}}=\sqrt{\frac{\pi}{24}}\left(\lambda_{N} / d\right)(R / d)^{1 / 4} .
$$


If $\gamma \gg 1,4 \pi \chi$ goes to the known value of $-3 / 4$ and the spatial profile of $A$ is determined by the magnetic energy. If $\gamma \ll 1$, the inhomogeneous terms dominate and $4 \pi \chi$ goes to -1 . The crossover takes place at $\gamma \simeq 1$.

Figure 5 shows the susceptibility as a function of the parameter $\gamma$ defined in Eq. (56). The universal behavior predicted by Eq. (51) and following (solid line) is compared to a full numerical solution of the integro-differential equation Eq. (37) for $R / d=10^{8}$ (symbols). The two curves agree perfectly for $\gamma \lesssim 5$; the deviation for larger values of $\gamma$ is explained by the fact that the condition $\lambda_{N} \ll d$ assumed in the derivation of the universal curve is not fulfilled. The susceptibility shows a very interesting behavior. For small values of $\gamma$ the susceptibility deviates substantially from the planar case. In particular, the absolute value of the susceptibility increases, i.e., the screening is enhanced by the cylindrical geometry. This is in contrast to the previously discussed behavior for $R \approx d$. The difference can be understood by looking at the magnetic field inside the normal metal, see Fig. 6. The spatially dependent integral kernel allows the existence of higher Fourier components of the current, instead of only the lowest-order component $j=$ const. as in the planar case. This leads to characteristic spatial oscillations of the magnetic field, which can show several sign changes inside the normal-metal layer. In particular, the field at the interior interface to the superconductor is also oscillating as a function of the parameter $\gamma$. It is interesting to note that a similar phenomenon occurs in the so-called Pippard superconductors, ${ }^{18}$ and has been experimentally observed. ${ }^{35}$

Finally, we would like to point out the qualitative similarity of the effect discussed and the reduced diamagnetic response found in Ref. 14 for a planar geometry and anomalously small impurity concentrations. In both cases, the origin of the effect is the absence of the contribution of very long trajectories. While in the cylindrical geometry this is due to the curvature of the normal-metal layer that leads to a typical length scale of these trajectories of $\sqrt{d R}(\gg d$ for $d \ll R$ ), the presence of impurities in the planar geometry limits the trajectory length to the elastic mean free path.

\section{CONCLUSION}

We have investigated the induced magnetic screening properties of cylindrical normal metal-superconductor heterostructures. The ballistic screening leads to a peculiar non-local current-vector potential relation inside the normal metal, governed by the parameter $\gamma$ defined in Eq. (56). For $\gamma>1$ the screening is like in the planar case, viz. the current density is constant, the magnetic field decays linearly down to $-H / 2$ and the 


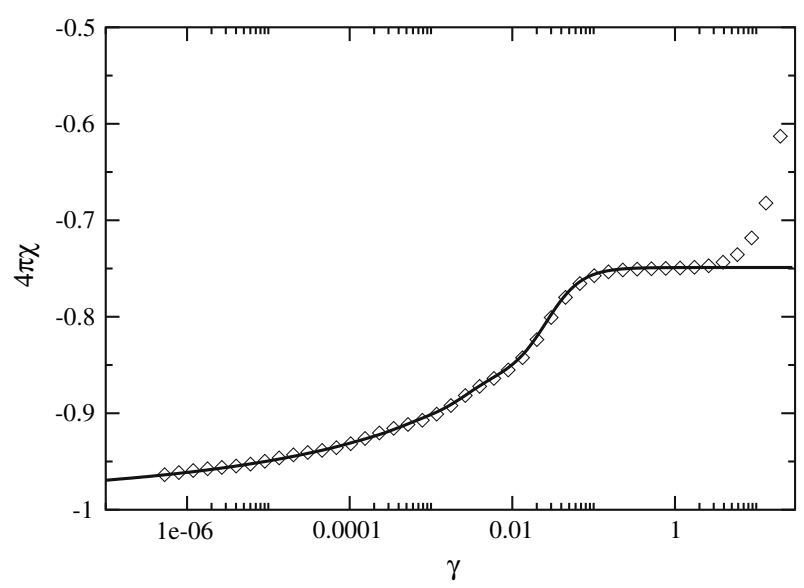

Fig. 5. Susceptibility as a function of the parameter $\gamma$ defined in Eq. (56). Solid line: universal behavior predicted by Eqs. (51)-(55). Symbols: solution of Eqs. (37), (25), (26), and (41) for $R / d=10^{8}$.

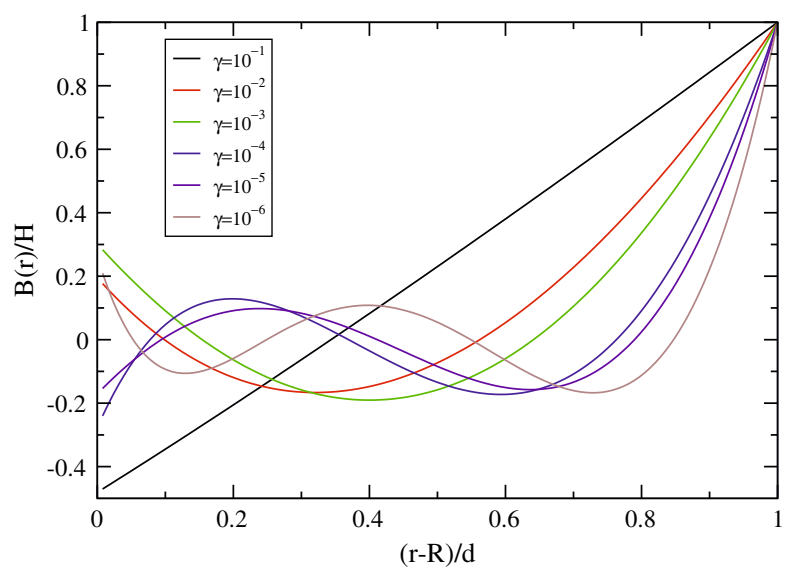

Fig. 6. (Color online) Magnetic field distribution in the normal layer for different values of $\gamma$.

susceptibility is $4 \pi \chi=-3 / 4$. In contrast, for $\gamma<1$, the field has an oscillatory spatial dependence, including several sign changes inside the normal metal. Overall, the magnetic susceptibility is enhanced and reaches the ideal diamagnetic value -1 in the limit $\gamma \ll 1$. 
This work was financially supported the SFB 513 Nanostructures at Surfaces and Interfaces of the DFG, the Swiss NSF, and the NCCR Nanoscience.

\section{REFERENCES}

1. P. G. de Gennes, Rev. Mod. Phys. 36, 225 (1964).

2. Orsay Group on Superconductivity, D. Brewer in Quantum Fluids, (ed.) North-Holland, Amsterdam, (1966).

3. A. C. Mota, D. Marek, and J. C. Weber, Helv. Phys. Acta. 55, 647 (1982).

4. Th. Bergmann, K. H. Kuhl, B. Schröder, M. Jutzler, and F. Pobell, J. Low. Temp. Phys. 66, 209 (1987)

5. A. C. Mota, P. Visani, and A. Pollini, J. Low. Temp. Phys. 76, 465 (1989).

6. P. Visani, A. C. Mota, and A. Pollini, Phys. Rev. Lett. 65, 1514 (1990).

7. A. C. Mota, P. Visani, A. Pollini, and K. Aupke, Physica B 197, 95 (1994).

8. H. Stalzer, A. Cosceev, C. Sürgers, and H. von Löhneysen, Europhys. Lett. 76, 121 (2006).

9. A. D. Zaikin, Solid State Commun. 41, 533 1982).

10. S. Higashitani and K. Nagai, J. Phys. Soc. Japan 64, 549 (1995).

11. H. Onoe, A. Sumuiyama, M. Nakagawa, and Y. Oda, J. Phys. Soc. Jpn. 64, 2138 (1995).

12. W. Belzig, C. Bruder, and G. Schön, Phys. Rev. B 53, 5727 (1996).

13. A. L. Fauchère and G. Blatter, Phys. Rev. B 56, 14102 (1997).

14. W. Belzig, C. Bruder, and A. L. Fauchère, Phys. Rev. B 58, 14531 (1998).

15. F. and H. London, Proc. Phys. Soc. Lond. A149, 71 (1938).

16. Y. Oda and H. Nagano, Solid State Commun. 35, 631 (1980).

17. A. Sumiyama, Y. Oda. and H. Nagano, J. Phys. Soc. Jpn. 53, 2449 (1984).

18. A. B. Pippard, Proc. R. Soc. Lond. Ser. A 216, 547 (1953).

19. F. B. Müller-Allinger and A. C. Mota, Phys. Rev. Lett. 84, 3161 (2000).

20. F. B. Müller-Allinger and A. C. Mota, Phys. Rev. B 62, R6120 (2000).

21. C. Bruder and J. Imry, Phys. Rev. Lett. 80, 5782 (1998). See also A. L. Fauchère, V. Geshkenbein, and G. Blatter, ibid. 82, 1796 (1999); and C. Bruder and J. Imry, ibid. 82, 1797 (1999).

22. A. Fauchère, W. Belzig, and G. Blatter, Phys. Rev. Lett. 82, 3336 (1999).

23. W. Belzig, Physica B 284, 1890 (2000).

24. F. Niederer, A. Fauchère, and G. Blatter, Phys. Rev. B 65, 132515 (2002).

25. W. Belzig, F. K. Wilhelm, C. Bruder, G. Schön, and A. D. Zaikin, Superlattices Microstructures 25, 1251 (1999).

26. F. B. Müller-Allinger, A. C. Mota, and W. Belzig, Phys. Rev. B 59, 8887 (1999).

27. M. Ashida, S. Aoyama, J. Hara, and K. Nagai, Phys. Rev. B 40, 8673 (1989).

28. O. Narikiyo and H. Fukuyama, J. Phys. Soc. Jpn. 58, 4557 (1989).

29. A. V. Galaktionov and A. D. Zaikin, Phys. Rev. B 65, 184507 (2002).

30. A. V. Galaktionov and A. D. Zaikin, Phys. Rev. B 67, 184518 (2003).

31. G. Eilenberger, Z. Phys. 214, 195 (1968).

32. A. I. Larkin and Yu. N. Ovchinnikov, Sov. Phys. JETP 26, 1200 (1968).

33. A. F. Andreev, Sov. Phys. JETP 22, 455 (1966).

34. A. D. Zaikin and G. F. Zharkov, Sov. Phys. JETP 54, 944 (1981).

35. K. E. Drangeid and R. Sommerhalder, Phys. Rev. Lett. 8, 467 (1962). 